\title{
Grand-design nuclear spiral as a signature of supermassive black hole
}

\author{
H. B. Ann $^{1}$ \\ ${ }^{1}$ Department of Earth Sciences, Pusan National University, Busan, 609-735, Korea \\ email: hbann@pusan.ac.kr
}

\begin{abstract}
From hydrodynamical simulations for the response of gaseous disks to imposed potentials, including those of a bar and supermassive black hole (SMBH), we found that the morphology of nuclear features developed by gas flows driven by the bar depends strongly on the sound speed of gas and the mass of SMBH which governs the potential shape in the central kiloparsec. Thus, nuclear features seem to provide a promising diagnostic for the inner dynamics of spiral galaxies. Specifically, a tightly wound grand-design nuclear spiral that winds more than $2 \pi$ indicates the presence of an SMBH which is massive enough to remove the IILR.
\end{abstract}

\section{Introduction}

The nuclear spirals are found to have a variety of morphologies including the granddesign nuclear spiral, which is thought to depend on the physical conditions in the central kiloparsec of galaxies. The gravitational potential shape of the nuclear region is believed to be much affected by the presence of a SMBH. However, the interplay between the $\mathrm{SMBH}$ and interstellar medium that leads to the formation of nuclear features such as nuclear spirals has not been studied much. Since nuclear spirals are found to be preponderant in active galaxies (Martini et al. 2003) and SMBH's are believed to be present in AGNs, we may expect some relationship between the nuclear spirals and SMBHs. The aim of the present study is to understand the dependence of the nuclear morphology on the mass of the SMBH $\left(M_{b h}\right)$ and the sound speed in the gas $\left(c_{s}\right)$.

\section{Models and Methods}

We adopted the PMSPH code of Fux (1999) for the simulation of the gas response to the imposed potentials with the assumption of an isothermal equation of state for the gas. We assumed fixed potentials for the halo, bulge, disk and bar following Ann \& Lee (2000). We also assumed a point mass potential for the SMBH. These potentials are fixed in the frame corotating with the bar. The self-gravity of the gas was included. The total visible mass of a model galaxy $\left(M_{G}\right)$ is assumed to be $(4 \sim 25) 10^{10} M_{\odot}$ and D/B (Disk to Bulge ratio) is taken as 2 for early type galaxies and 5 for late type ones. We considered SMBHs whose masses are $(0 \sim 0.01) M_{G}$. We assumed moderately strong bars that have fractional mass of 0.2 and axial ratio of 3 . The pattern speeds of bars were selected to make bars end near the corotation radii. The sound speeds of gas were varied between 5 $\mathrm{km} \mathrm{s}^{-1}$ and $35 \mathrm{~km} \mathrm{~s}^{-1}$. Although our code is a fully three-dimensional one, we confined the present simulations to two-dimensional ones to increase the spatial resolution with a limited number of particles as $\mathrm{N}=20,000$. 




Figure 1. Snapshots of the evolution of gaseous disks in three models for late type galaxies. All three models assume central SMBH with $M_{b h}=0.01 M_{G}$. The snapshots are taken at $10 \mathrm{bar}$ rotation time and the unit of length is kpc. (left) $M_{G}=4 \cdot 10^{10} M_{\odot}, B / D=5, c_{s}=15 \mathrm{~km} \mathrm{~s}^{-1}$; (middle) $M_{G}=2.3 \cdot 10^{11} M_{\odot}, B / D=5, c_{s}=15 \mathrm{~km} \mathrm{~s}^{-1} ; \quad$ (right) $M_{G}=2.3 \cdot 10^{11} M_{\odot}, B / D=5, c_{s}=30 \mathrm{~km} \mathrm{~s}^{-1}$.

\section{Results}

The evolution of the nuclear regions of gaseous disks driven by the bar depends on the sound speed in the gas as well as on the central mass concentration, caused by the $\mathrm{SMBH}$ and the global mass distribution. The formation of tightly wound nuclear spirals with large winding angles $(\gtrsim 2 \pi)$ requires some specific physical conditions, i.e., i) a massive central black hole is necessary to remove the IILR, ii) the sound speeds in the gas are high enough to allow sufficient mass inflow toward the center but they are low enough to keep the principal shock as an off-axis shock (Englemaier \& Gerhard 1997). The optimum sound speed for the formation of tightly wound nuclear spirals that have large winding angles depends on the global mass distribution as well as the mass of the $\mathrm{SMBH}$. For example, $c_{s} \approx 15 \mathrm{~km} \mathrm{~s}^{-1}$ is the most plausible sound speed for the models of $M_{G} \sim 4 \times 10^{10} M_{\odot}$ and $\mathrm{D} / \mathrm{B}=5$, while $c_{s} \approx 30 \mathrm{~km} \mathrm{~s}^{-1}$ is more plausible sound speed for the models of $M_{G} \sim 2.3 \times 10^{11} M_{\odot}$ and $\mathrm{D} / \mathrm{B}=5$ (see Fig. 1). The open grand-design nuclear spirals of which winding angles are less than $\sim \pi$ are likely to be formed in the models with large D/B when the mass of SMBHs is small enough to allow two ILRs and the interstellar medium has proper sound speeds. In general, lower sound speed leads to the formation of ring-like nuclear spirals similar to that in the middle panel of Fig. 1. Thus, nuclear spirals seem to provide a promising diagnostic for the dynamics of the central kiloparsec of spiral galaxies.

\section{Acknowledgements}

HBA thank to Roger Fux for the PMSPH code available and Hyesung Kang for the help in setting up the code for the present simulations. This work was supported in part by ARCSEC and by grant No. R01-1999-00023 from KOSEF. Most of the computations are performed using the super computer facilities in KISTI.

\section{References}

Ann, H. B., \& Lee, H. M. 2000, J. Korean Astronomical Soc., 33, 1

Englmaier, P., \& Gerhard, O. 1997, MNRAS, 287, 57

Fux, R. 1999, A\&A, 345, 787

Martini, P., Regan, M. W., Mulchaey, J. S., \& Pogge, R., 2003, ApJS, 146, 353 